\title{
Un Mousquetaire du journalisme: Alexandre Dumas, dir. S. MOMBERT et C. SAMINADAYAR-PERRIN
}

\section{Angels Santa}

\section{(2) OpenEdition}

\section{Journals}

Édition électronique

URL : https://journals.openedition.org/studifrancesi/45264

DOI : 10.4000/studifrancesi.45264

ISSN : 2427-5856

Éditeur

Rosenberg \& Sellier

Édition imprimée

Date de publication : 1 août 2021

Pagination : 387-389

ISSN : 0039-2944

\section{Référence électronique}

Angels Santa, « Un Mousquetaire du journalisme: Alexandre Dumas, dir. s. mombert et c. saminadaYAR-

PERRIN », Studi Francesi [En ligne], 194 (LXV | II) | 2021, mis en ligne le 01 septembre 2021, consulté le 15 octobre 2022. URL : http://journals.openedition.org/studifrancesi/45264 ; DOI : https://doi.org/ 10.4000/studifrancesi.45264

Ce document a été généré automatiquement le 15 octobre 2022.

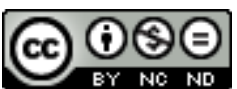

Creative Commons - Attribution - Pas d'Utilisation Commerciale - Pas de Modification 4.0 International - CC BY-NC-ND 4.0

https://creativecommons.org/licenses/by-nc-nd/4.0/ 


\title{
Un Mousquetaire du journalisme: Alexandre Dumas, dir. S. MOMBERT et C.
} SAMINADAYAR-PERRIN

\author{
Angels Santa
}

\section{RÉFÉRENCE}

Un Mousquetaire du journalisme: Alexandre Dumas, dir. S. MOMBERT et C. SAMINADAYAR-PERRIN, Besançon, Presses universitaires de Franche-Comté, 2019, 250 pp.

1 Aussi bien Sarah Mombert que Corinne Saminadayar-Perrin ont décidé de nous présenter un Dumas moins populaire que celui qui a écrit les romans que nous connaissons tous, un Dumas plus quotidien et plus lié à l'actualité: le Dumas journaliste.

Les deux auteures ont beaucoup travaillé dans le domaine de la presse. Sarah Mombert est maître de conférences en littérature française du XIX ${ }^{\mathrm{e}}$ siècle à l'École Normale Supérieure de Lyon; elle s'est intéressée particulièrement au roman et à la presse dans une perspective d'histoire littéraire et culturelle. Elle a dirigé l'édition en ligne des journaux dirigés par Alexandre Dumas et a publié d'importants travaux sur le sujet comme le livre Entre presse et littérature. «Le Mousquetaire», journal de M. Alexandre Dumas avec Pascal Durant en 2009 aux Presses Universitaires de Liège. Le livre qui nous occupe est une suite de ce travail dont il élargit la portée. Corinne Saminadayar-Perrin est professeure à l'Université de Montpellier 3 et s'intéresse aux rapports entre presse et littérature au XIX ${ }^{\mathrm{e}}$ siècle. Elle a publié sur ce sujet en 2007 Les Discours du journal: rhétorique et médias au XIX ${ }^{\mathrm{e}}$ siècle (1836-1885), aux Publications de l'université de SaintÉtienne et le livre qui nous occupe se situe au centre de la question.

3 Cet ouvrage collectif réalise un parcours assez exhaustif sur l'œuvre journalistique dumasienne en tenant compte des différentes étapes successives. Il comporte trois parties précédées par une introduction et suivies d'une conclusion. La première partie analyse le période de 1848, quand Dumas publie "Le Mois". Dans la deuxième on nous 
présente "Le Mousquetaire" (1853-1857) et "Le Monte-Cristo" (1857-1860) ainsi que les différents avatars de la création de l'écrivain. Dans la dernière partie, beaucoup plus courte, le centre d'intérêt se déplace à "L'Indipendente" (1860-1864) qui voit le jour à Naples pour appuyer la geste de Garibaldi. Le livre est complété par un appareil critique remarquable: une bibliographie en trois volets (sources de bibliographie générale, éléments de bibliographie et bibliographie des œuvres de Dumas) et deux index très utiles et précis: un index nominum et un index des œuvres littéraires et artistiques et des périodiques, ce qui accorde à la publication une valeur supplémentaire.

Dans l'introduction Corinne SAMINADAYAR-PERRIN (Alexandre Dumas, mousquetaire du journalisme, pp. 7-18) nous présente un Dumas tenté par la portée du journalisme: «dès son entrée dans la vie littéraire le jeune dramaturge à succès se taille dans et par la presse un espace à la mesure [...] d'une personnalité qui se veut publique, dans tous les sens du terme» (p. 7). Il est présenté comme un «infatigable militant du romantisme» (p. 8), ce qui lui permet de défendre l'idéal d'une littérature populaire démocratique et interactive. Elle nous montre comme il devient très tôt, dès 1929, un habituel des journaux, avec les critiques qu'il publie à "L'impartial" et à "La Presse" en devenant le critique des critiques. À partir de là, elle trace le parcours réalisé par les contributeurs du livre pour étudier les différentes facettes du travail dumasien par rapport au journalisme et à ses alentours. Elle aboutit à l'idée que le «journalisme représente pour Dumas l'une des dimensions constitutives du journalisme à l'ère médiatique» (p. 18), en concluant qu'il «invente une nouvelle façon d'être écrivain, parfois hors le livre et sans le livre, par le discours autant que par le texte, dans la vie comme dans la création» (p. 18).

5 La première partie s'intitule «1848: écrire et faire l'histoire». Elle est introduite par Vincent ROBERT (Dumas parmi d'autres: romancier, journaliste et journaux en 1848, pp. 21-31) qui tente de nous expliquer que le monde des hommes de lettres et celui des journalistes n'étaient pas si distincts à l'époque. La plupart des romanciers s'étaient introduits dans la presse, leur collaboration s'était renforcée à partir de 1830 au moment de l'apparition du roman feuilleton. En 1848 les écrivains auront des problèmes et beaucoup vont créer leur propre journal. Seulement Dumas et Hugo eurent du succès avec "Le Mois" (mensuel) et le "L'Événement". Nous voyons que la presse pendant la Deuxième République n'échappait pas à un procès de spécialisation, ce qui explique ce phénomène. Sébastien HALLADE (Alexandre Dumas, un journaliste engagé sous la Deuxième République, pp. 33-46) explique l'entreprise de la création du "Mois", entreprise qui va durer deux ans et qui va soutenir l'action politique engagée de l'écrivain; le journal disparaît pour des raisons financières, car Dumas avait beaucoup de dettes. Il faisait tout dans le journal, sa finalité fondamentale était d'éclairer le peuple. Cependant il ne réussit pas à se faire reconnaître comme prophète du peuple mais son travail annonce l'écrivain engagé de la deuxième moitié du siècle. Corinne SAMINADAYAR-PERRIN continue l'étude de ce journal ("Le Mois" (1848). Paradoxe d'une histoire immédiate, pp.47-62) en montrant comment l'année 1848 fut une année catastrophique pour Dumas qui ne retrouvera plus l'influence eue sous la monarchie de juillet. Son expérience comme romancier historique lui sert pour confectionner son journal et il utilisera les mêmes techniques narratives que dans l'œuvre romanesque, ce qui lui permet de maintenir son lecteur en suspens en offrant un texte hybride qui va de l'histoire à la fiction. Charles GRIVEL (Alexandre Dumas: la réalité de tous les mois, pp. 63-76) montre l'omniprésence de Dumas dans le journal, il y est au centre, il fait 
tout, il remplit tous les rôles et domine d'une façon panoramique l'existence de la publication comme s'il s'agissait de son univers romanesque. Ludovic FRAUBERT (Alexandre Dumas et les doctrines socialistes de 1848, pp.77-85) analyse la position idéologique de l'écrivain aussi bien dans le journal qu'il a fondé "Le Mois" que dans les journaux auxquels il collabore comme "Le Constitutionnel", "La Patrie", "La Liberté", etc. En prenant comme point de départ Le Comte de Monte-Cristo il aborde le problème de la distribution de richesses et de la propriété. Dumas n'a pas une opinion unanime sur les diverses positions socialistes et il pense que le progrès amènera inévitablement une guerre civile.

6 La deuxième partie porte comme titre: «Causeries, mémoires et fictions». Le premier article, celui de Maria Lucia DIAS MENDES («Mes Mémoires» dans "Le Mousquetaire”. La voix du narrateur, pp. 89-96) aborde l'écriture des mémoires de Dumas, qu'il envisage à l'âge de 45 ans; l'ouvrage va refléter les changements de vie subis par l'auteur pendant la période de sa rédaction, entre 1847 et 1855. Le journal "La Presse" va se charger d'en publier la plus grande partie jusqu'en octobre 1853 où Dumas est prévenu par Girardin qu'il a décidé d'interrompre la publication. Il décide alors de la poursuivre dans "Le Mousquetaire" en essayant d'épouser la dynamique du journal. Sarah MOMBERT ("Le Moustiquaire", journal parodique $d u$ "Mousquetaire", pp.97-110) analyse "Le Moustiquaire", journal pastiche du "Mousquetaire" qui en fait la parodie. En principe très critique, il devient en fait une exaltation de Dumas et contribue à son succès. Mathias HAUSMANN («Les Ruines de Paris». Une forme particulière de littérature d'anticipation, pp. 111-121) s'intéresse à un texte humoristique «qui aura une influence importante sur une forme particulière de la littérature d'anticipation du XIx ${ }^{\mathrm{e}}$ siècle en France» (p. 111). Il va être la source d'inspiration de Joseph Méry, d'Alfred Bonnardot et d'Alfred Franklin. Hausmann montre la pertinence de ce thème récurrent, mis en valeur grâce aux Causeries dumasiennes. Cyrille FRANçoIs (Les contes $d u$ «Monte-Cristo». Alexandre Dumas et ses "Contes pour les grands et les petits enfants", pp.123-135) parle des contes publiés par l'écrivain qui représentent une dimension méconnue de son œuvre. Andersen, Grimm, Crowquill, Bechstein sont les modèles. Il travaille avec les traducteurs et des collaborateurs mais il apporte sa touche personnelle, c'est qui en fait une œuvre différente et originelle. À son tour, Julie ANSELMINI examine la question du merveilleux (Le merveilleux à l'épreuve du journal. L'exemple du "Mousquetaire", pp. 137-148) en se rapportant non seulement à Dumas mais surtout à ses collaborateurs dans le journal. Dans ces contes le surnaturel n'est pas aussi naturellement admis que dans ce type d'ouvrages; sans doute cela est dû à l'influence du journal et à l'influence du rationalisme moderne. Malgré cela, les textes réhabilitent le merveilleux et en cela "Le Mousquetaire" demeure foncièrement romantique.

7 La troisième partie «Un militant du romantisme à l'ère médiatique» réunit les contributions d'Isabelle SAFA (Dumas journaliste romancier, la geste de Garibaldi, pp. 151-163) et d'Alvio PATIERNo (La lutte éditoriale d'Alexandre Dumas contre le brigandage dans "L'Indipendente", pp. 165-177) qui s'occupent de l'aventure italienne de l'auteur. Safa montre comment Dumas met son art au service de Garibaldi pour le mythifier en héros en utilisant les méthodes du roman populaire qui lui permettent de donner au chef italien une véritable dimension politique. Patierno met en valeur la mission civilisatrice accordée par son directeur à "L'Indipendente", lutter contre «la Camorra, la Consorteria, la Camarilla» (p. 165). Il s'agit de la lutte contre le brigandage, en montrant l'engagement du citoyen contre les puissants. 
8 À Sandrine CARAVALHOSA (L'invention du journalisme autocentré, pp. 179-194) revient la tâche de la conclusion. Elle essaie de définir la littérature autocentrée, en montrant que la grande innovation de Dumas se trouve dans sa position romantique sur le domaine médiatique. Il est un écrivain très moderne; à l'époque actuelle il occuperait une première place «à la pointe des médias et des réseaux sociaux» (p. 194).

9 Le volume nous offre une présentation très large de la tâche journalistique de Dumas, en insistant sur toutes ses variantes et possibilités. Il est d'une grande richesse pour les études dumasiennes et deviendra un ouvrage incontournable pour tous ceux qui désirent approfondir le rôle des journaux dans la vie de l'auteur. 